\title{
Real-Time Large Displacement Elasticity for Surgery Simulation: Non-linear Tensor-Mass Model
}

\author{
G. Picinbono, H. Delingette, and N. Ayache \\ INRIA Sophia - Epidaure Project \\ 2004 Route des Lucioles BP 93 \\ 06902 Sophia Antipolis Cedex, France \\ \{Guillaume.Picinbono, Herve.Delingette, Nicholas.Ayache\}@sophia.inria.fr \\ http://www-sop.inria.fr/epidaure/Epidaure-eng.html
}

\begin{abstract}
In this paper, we describe the latest developments of the minimally invasive hepatic surgery simulator prototype developed at INRIA. A key problem with such a simulator is the physical modeling of soft tissues. We propose a new deformable model based on non-linear elasticity and the finite element method. This model is valid for large displacements, which means in particular that it is invariant with respect to rotations. This property improves the realism of the deformations and solves the problems related to the shortcomings of linear elasticity, which is only valid for small displacements. We also address the problem of volume variations by adding to our model incompressibility constraints. Finally, we demonstrate the relevance of this approach for the real-time simulation of laparoscopic surgical gestures on the liver.
\end{abstract}

\section{Introduction}

A major and recent evolution in abdominal surgery has been the development of laparoscopic surgery. In this type of surgery, abdominal operations such as hepatic resection are performed through small incisions. A video camera and special surgical tools are introduced into the abdomen, allowing the surgeon to perform a procedure less invasive. A drawback of this technique lies essentially in the need for more complex gestures and in the loss of direct visual and tactile information. Therefore the surgeon needs to learn and adapt himself to this new type of surgery and in particular to a new type of hand-eye coordination. In this context, surgical simulation systems could be of great help in the training process of surgeons.

Among the several key problems in the development of a surgical simulator $[1,13]$, the geometrical and physical representation of human organs remain the most important. The deformable model must be at the same time very realistic (both visually and physically) and very efficient to allow real-time deformations. Several methods have been proposed: spring-mass models $[8,12]$, free form deformations [2], linear elasticity with finite volume method [9] or various finite element methods $[6,10,15,4]$. 
In this article we propose a new real-time deformable model based on nonlinear elasticity and a finite element method. We first introduce the linear elasticity theory and its implementation through the finite element method, and we then highlight its shortcomings when the "small displacement" hypothesis does not hold. Then we focus on our implementation of St Venant-Kirchhoff elasticity and incompressibility constraints.

\section{Shortcomings of the Linear Elasticity}

Linear elasticity is often used for the modeling of deformable materials, mainly because the equations remain quite simple and the computation time can be optimized.

The physical behavior of soft tissue may be considered as linear elastic if its displacement and deformation remain small [11,14] (typically less than $10 \%$ of the mesh size). We represent the deformation of a volumetric model from its rest shape $\mathcal{M}_{\text {initial }}$ with a displacement vector $\mathbf{U}(x, y, z)$ for $(x, y, z) \in \mathcal{M}_{\text {initial }}$ and we write $\mathcal{M}_{\text {deformed }}=\mathcal{M}_{\text {initial }}+\mathbf{U}(x, y, z)$.

From this displacement vector, we define the linearized Green-St Venant strain tensor $(3 \times 3$ symmetric matrix $) E_{l}$ and its principal invariants $l_{1}$ and $l_{2}$ :

$$
E_{l}=\frac{1}{2}\left(\nabla \mathbf{U}+\nabla \mathbf{U}^{t}\right) \quad l_{1}=\operatorname{tr} E_{l} \quad l_{2}=\operatorname{tr} E_{l}^{2} .
$$

The linear elastic energy $W_{\text {Elastic }}$, for homogeneous isotropic materials, is defined by the following formula (see [5]):

$$
W_{\text {Elastic }}=\frac{\lambda}{2}\left(\operatorname{tr} E_{l}\right)^{2}+\mu \operatorname{tr} E_{l}^{2}=\frac{\lambda}{2}(\operatorname{div} \mathbf{U})^{2}+\mu\|\nabla \mathbf{U}\|^{2}-\frac{\mu}{2}\|\operatorname{rot} \mathbf{U}\|^{2},
$$

where $\lambda$ and $\mu$ are the Lam coefficients characterizing the material stiffness.

Equation 2, known as Hooke's law, shows that the elastic energy of a deformable object is a quadratic function of the displacement vector.

\subsection{Finite Element Method}

Finite element method is a classical way to solve linear elasticity problems. Its most interesting property is to provide a continuous description of physical equations. We chose to use $P_{1}$ finite elements where the elementary volume is a tetrahedron with a node defined at each vertex. At each point $\mathbf{M}(x, y, z)$ inside tetrahedron $\mathbf{T}_{i}$, the displacement vector is expressed as a function of the displacements $\mathbf{U}_{k}$ of vertices $\mathbf{P}_{k}$. For $P_{1}$ finite elements, interpolation functions $\Lambda_{k}$ are linear $\left(\left\{\Lambda_{k} ; k=0, \ldots, 3\right\}\right.$ are the barycentric coordinates of $\mathbf{M}$ in the tetrahedron): 


$$
\begin{aligned}
& \mathbf{U}(x, y, z)=\sum_{j=0}^{3} \mathbf{U}_{j} \Lambda_{j}(x, y, z) \quad \Lambda_{j}(x, y, z)=\alpha_{j} . X+\beta_{j} \\
& \alpha_{j}=\frac{(-1)^{j}}{6 V\left(\mathbf{T}_{i}\right)}\left(\mathbf{P}_{j+1} \times \mathbf{P}_{j+2}+\mathbf{P}_{j+2} \times \mathbf{P}_{j+3}+\mathbf{P}_{j+3} \times \mathbf{P}_{j+1}\right) \\
& \text { where } \times \text { stands for the cross product between two } \\
& \text { vectors, and } V\left(\mathbf{T}_{i}\right) \text { is the volume of the tetrahedron. }
\end{aligned}
$$

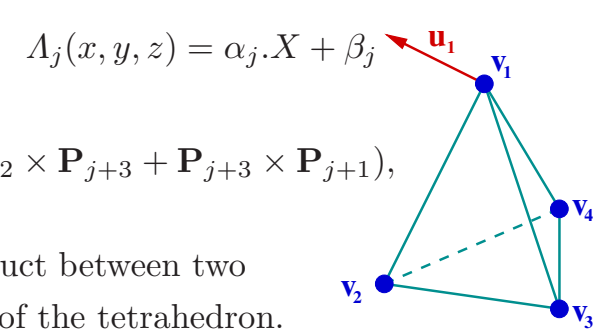

Fig. 1. $P_{1}$ finite element

Using this equation for the displacement vector $\mathbf{U}$ leads to the finite element formulation of linear elastic energy in the tetrahedron $\mathbf{T}_{i}[10]$ :

$$
\begin{aligned}
W_{\text {Elastic }}\left(\mathbf{T}_{i}\right) & =\sum_{j, k=0}^{3} \mathbf{U}_{j}^{t}\left[\mathcal{B}_{j k}^{\mathbf{T}_{i}}\right] \mathbf{U}_{k} \\
\mathcal{B}_{j k}^{\mathbf{T}_{i}} & =\lambda\left(\alpha_{j} \otimes \alpha_{k}\right)+\mu\left[\left(\alpha_{k} \otimes \alpha_{j}\right)+\left(\alpha_{j} . \alpha_{k}\right) I d_{3}\right],
\end{aligned}
$$

where $\left[\mathcal{B}_{j k}^{\mathbf{T}_{i}}\right]$ is the tetrahedron contribution to the stiffness tensor of the edge $\left(\mathbf{P}_{j}, \mathbf{P}_{k}\right.$ ) (or of the vertex $\mathbf{P}_{j}$ if $j=k$ ), $\left\{\alpha_{j}, k=0, . ., 3\right\}$ are the shape vectors of the tetrahedron and $\otimes$ stands for the tensor product of two vectors.

Finally, to obtain the force $\mathbf{F}_{p}^{\mathbf{T}_{i}}$ applied by the tetrahedron $\mathbf{T}_{i}$ on the vertex $\mathbf{P}_{p}$, we derive the elastic energy with respect to the vertex displacement $\mathbf{U}_{p}$ :

$$
\mathbf{F}_{p}^{\mathbf{T}_{i}}=2 \sum_{j=0}^{3}\left[\mathcal{B}_{p j}^{\mathbf{T}_{i}}\right] \mathbf{U}_{j} .
$$

We have been using this linear elasticity formulation for several years through two deformable models, the pre-computed model [6] and the tensor-mass model $[10,7]$. Furthermore, it can be extended to anisotropic linear elasticity [15], which allows to model fiber-reinforced materials, very common within biological tissues (blood vessels, tendons, muscles, ...).

\subsection{The Problem of Rotational Invariance}

The main limitation of the linear model is that it is not invariant with respect to rotations. When the object undergoes a rotation, the elastic energy increases, leading to a variation of the volume (see figure 2). In the case of a global rotation of the object, we could solve the problem with a specific change of the reference frame.

But this solution proves itself to be ineffective when only one part of the object undergoes a rotation (which is the case in general). This case is presented by the cylinder of figure 3: the bottom face is fixed and a force is applied to the central top vertex. Arrows show the trajectory of some vertices, which are constrained by the linear model to move along straight lines. This results in the 

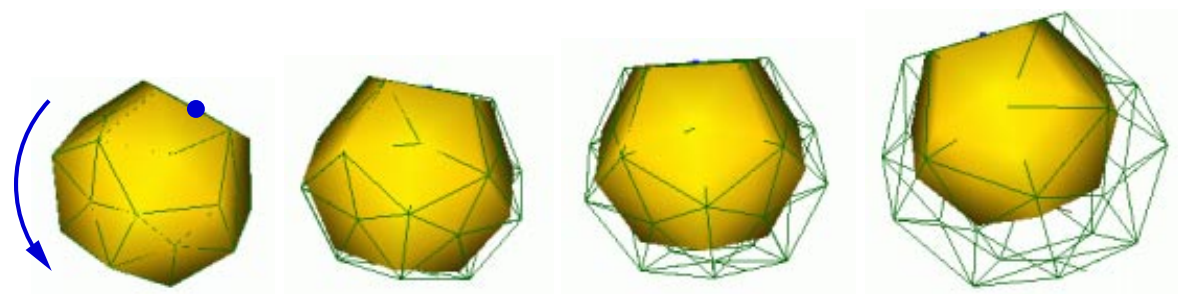

Fig. 2. Global rotation of the linear elastic model (wire-frame)

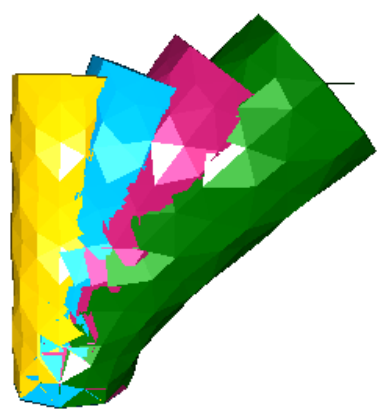

(a)

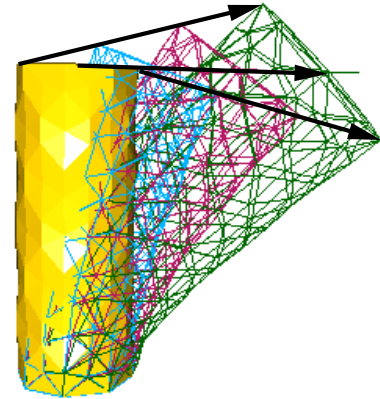

(b)

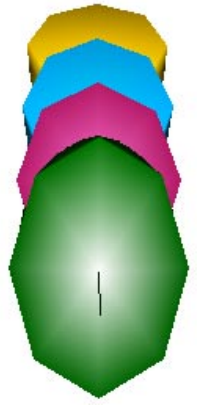

(c)

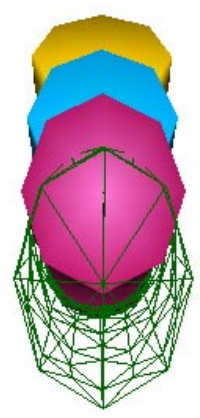

(d)

Fig. 3. Successive deformations of a linear elastic cylinder. (a) and (b): side view. (c) and (d): top view

distortion of the mesh. Furthermore, this abnormal deformation is anisotropic since the object only deforms itself in the rotation plane (figure 3(c) and 3(d)).

This unrealistic behaviour of the linear elastic model for large displacements leads us to consider different models of elasticity.

\section{St Venant-Kirchhoff Elasticity}

A model of elasticity is considered as a large displacement model if it derives from a strain tensor which is a quadratic function of the deformation gradient. Most common tensors are the left and right Cauchy-Green strain tensors (respectively $B=\nabla \phi \nabla \phi^{t}$ and $C=\nabla \phi^{t} \nabla \phi, \phi$ being the deformation).

The St Venant-Kirchhoff model is a generalization of the linear model for large displacements, and is a particular case of hyperelastic materials. The basic energy equation is the same (equation 2), but now $E$ stands for the complete Green-St Venant strain tensor:

$$
E=\frac{1}{2}(C-I)=\frac{1}{2}\left(\nabla \mathbf{U}+\nabla \mathbf{U}^{t}+\nabla \mathbf{U}^{t} \nabla \mathbf{U}\right) .
$$


Elastic energy, which was a quadratic function of $\nabla \mathbf{U}$ in the linear case, is now a polynomial of order four with respect to $\nabla \mathbf{U}$ :

$$
\begin{aligned}
W & =\frac{\lambda}{2}\left[(\operatorname{div} \mathbf{U})+\frac{1}{2}\|\nabla \mathbf{U}\|^{2}\right]^{2}+\mu\|\nabla \mathbf{U}\|^{2}-\frac{\mu}{2}\|\operatorname{rot} \mathbf{U}\|^{2} \\
& +\mu\left(\nabla \mathbf{U}: \nabla \mathbf{U}^{t} \nabla \mathbf{U}\right)+\frac{\mu}{4}\left\|\nabla \mathbf{U}^{t} \nabla \mathbf{U}\right\|^{2},
\end{aligned}
$$

where $\quad A: B=\operatorname{tr}\left(A^{t} B\right)=\sum_{i, j} a_{i j} b_{i j} \quad$ is the dot product of two matrices.

\subsection{Finite Element Modeling}

With the notations introduced in section 2.1, we express the St Venant-Kirchhoff elastic model with finite element theory as:

$$
\begin{aligned}
W\left(\mathbf{T}_{i}\right) & =\sum_{j, k} \mathbf{U}_{j}^{t}\left[\mathcal{B}_{j k}^{\mathbf{T}_{i}}\right] \mathbf{U}_{k}+\sum_{j, k, l}\left(\mathbf{U}_{j} \cdot \mathcal{C}_{j k l}^{\mathbf{T}_{i}}\right)\left(\mathbf{U}_{k} \cdot \mathbf{U}_{l}\right) \\
& +\sum_{j, k, l, m} \mathcal{D}_{j k l m}^{\mathbf{T}_{i}}\left(\mathbf{U}_{j} \cdot \mathbf{U}_{k}\right)\left(\mathbf{U}_{l} \cdot \mathbf{U}_{m}\right)
\end{aligned}
$$

where:

$-\mathcal{B}_{j k}^{\mathbf{T}_{i}}$ is the (3x3) symmetric matrix of the linear elastic model (equation 3$)$,

$-\mathcal{C}_{j k l}^{\mathbf{T}_{i}}$ is a vector: $\mathcal{C}_{j k l}^{\mathbf{T}_{i}}=\frac{\lambda}{2} \alpha_{j}\left(\alpha_{k} \cdot \alpha_{l}\right)+\frac{\mu}{2}\left[\alpha_{l}\left(\alpha_{j} . \alpha_{k}\right)+\alpha_{k}\left(\alpha_{j} \cdot \alpha_{l}\right)\right]$,

- and $\mathcal{D}_{j k l m}^{\mathbf{T}_{i}}$ is a scalar: $\mathcal{D}_{j k l m}^{\mathbf{T}_{i}}=\frac{\lambda}{8}\left(\alpha_{j} . \alpha_{k}\right)\left(\alpha_{l} . \alpha_{m}\right)+\frac{\mu}{4}\left(\alpha_{j} . \alpha_{m}\right)\left(\alpha_{k} . \alpha_{l}\right)$.

The force applied at each vertex inside a tetrahedron is derived from the elastic energy $W\left(\mathbf{T}_{i}\right)$ :

$$
\begin{aligned}
\mathbf{F}^{p}\left(\mathbf{T}_{i}\right) & =\underbrace{2 \sum_{j}\left[\mathcal{B}_{p j}^{\mathbf{T}_{i}}\right] \mathbf{U}_{j}}_{\mathbf{F}_{1}^{p}\left(\mathbf{T}_{i}\right)}+\underbrace{\sum_{j, k} 2\left(\mathbf{U}_{k} \otimes \mathbf{U}_{j}\right) \mathcal{C}_{j k p}^{\mathbf{T}_{i}}+\left(\mathbf{U}_{j} \cdot \mathbf{U}_{k}\right) \mathcal{C}_{p j k}^{\mathbf{T}_{i}}}_{\mathbf{F}_{2}^{p}\left(\mathbf{T}_{i}\right)} \\
+ & \underbrace{4 \sum_{j, k, l} \mathcal{D}_{j k l p}^{\mathbf{T}_{i}} \mathbf{U}_{l} \mathbf{U}_{k}^{t} \mathbf{U}_{j}}_{\mathbf{F}_{3}^{p}\left(\mathbf{T}_{i}\right)} .
\end{aligned}
$$

The first term of the elastic force $\left(\mathbf{F}_{1}^{p}\left(\mathbf{T}_{i}\right)\right)$ corresponds to the linear elastic case presented in section 2.1. The next part of the article deals with the generalization of the tensor-mass model to large displacements.

\subsection{Non-linear Tensor-Mass Model}

The main idea of the tensor-mass model is to split, for each tetrahedron, the force applied at a vertex in two parts: a force created by the vertex displacement and forces produced by the displacements of its neighbours:

$$
\mathbf{F}_{1}^{p}\left(\mathbf{T}_{i}\right)=\left[\mathcal{B}_{p p}^{\mathbf{T}_{i}}\right] \mathbf{U}_{p}+\sum_{j \neq p}\left[\mathcal{B}_{p j}^{\mathbf{T}_{i}}\right] \mathbf{U}_{j}
$$


This way we can define for each tetrahedron a set of local stiffness tensors for vertices $\left(\left\{\mathcal{B}_{p p}^{\mathbf{T}_{i}} ; p=0, \ldots, 3\right\}\right)$ and for edges $\left(\left\{\mathcal{B}_{p j}^{\mathbf{T}_{i}} ; p, j=0, \ldots, 3 ; p \neq j\right\}\right)$. By doing this for every tetrahedron, we can accumulate on vertices and edges of the mesh the corresponding contributions to the global stiffness tensors:

$$
\mathcal{B}_{p p}=\sum_{\mathbf{T}_{i} \in N\left(\mathbf{V}_{p}\right)} \mathcal{B}_{p p}^{\mathbf{T}_{i}} \quad \mathcal{B}_{p j}=\sum_{\mathbf{T}_{i} \in N\left(\mathbf{E}_{p j}\right)} \mathcal{B}_{k l}^{\mathbf{T}_{i}} .
$$

These stiffness tensors are computed when creating the mesh and are stored for each vertex and edge of the mesh.

The same principle can be applied to the quadratic term $\left(\mathbf{F}_{2}^{p}\left(\mathbf{T}_{i}\right)\right.$ of equation 8 ) and the cubic term $\left(\mathbf{F}_{3}^{p}\left(\mathbf{T}_{i}\right)\right)$. The former brings stiffness vectors for vertices, edges, and triangles, and the latter brings stiffness scalars for vertices, edges, triangles, and tetrahedra. The following array (table 1) summarizes the stiffness data stored on each geometrical primitive of the mesh:

\begin{tabular}{|c|c|c|c|}
\hline Stiffness data distribution & Tensors & Vectors & Scalars \\
\hline \begin{tabular}{|c|} 
Vertex $\mathbf{V}_{p}$ \\
\end{tabular} & $\mathcal{B}^{p p}$ & $\mathcal{C}^{p p p}$ & $\mathcal{D}^{p p p p}$ \\
\hline Edge $\mathbf{E}_{p j}$ & $\mathcal{B}^{p j}$ & $\begin{array}{ll}\mathcal{C}^{p p j} & \mathcal{C}^{j p p} \\
\mathcal{C}^{j j p} & \mathcal{C}^{p j j} \\
\end{array}$ & $\begin{array}{ll}\mathcal{D}^{j p p p} & \mathcal{D}^{j j j p} \mathcal{D}^{j p j p} \\
\mathcal{D}^{p j j p} & \mathcal{D}^{j j p p}\end{array}$ \\
\hline Triangle $\mathbf{F}_{p j k}$ & & $\begin{array}{l}\mathcal{C}^{j k p} \\
\mathcal{C}^{k j p} \\
\mathcal{C}^{p j k}\end{array}$ & $\begin{array}{lll}\mathcal{D}^{j k p p} & \mathcal{D}^{j p k p} & \mathcal{D}^{p j k p} \\
\mathcal{D}^{j j k p} & \mathcal{D}^{j k j p} & \mathcal{D}^{k j j p} \\
\mathcal{D}^{k k j p} & \mathcal{D}^{k j k p} & \mathcal{D}^{j k k p}\end{array}$ \\
\hline Tetrahedron $\mathbf{T}_{p j k l}$ & & & 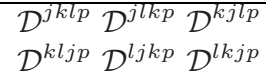 \\
\hline
\end{tabular}

Table 1. Storage of the stiffness data on the mesh

Given a tetrahedral mesh of a solid - in our case an anatomical structure we build a data structure incorporating the notion of vertices, edges, triangles, and tetrahedra, with all the necessary neighbours. For each vertex, we store its current position $\mathbf{P}_{p}$, its rest position $\mathbf{P}_{p}^{0}$, and its stiffness data. For each edge, we store stiffness data. Finally for each tetrahedron, we store the Lam coefficients $\lambda$ and $\mu$, the four shape vectors $\alpha_{k}$, and the stiffness data.

During the simulation, we compute forces for each vertex, edge, triangle, and tetrahedron, and we update the vertex positions from the differential equations of continuum mechanics [3]:

$$
\mathbf{M U ̈}+\mathbf{C U ்}+\mathbf{F}(\mathbf{U})=\mathbf{R} .
$$

Following finite element theory, the mass $\mathbf{M}$ and damping $\mathbf{C}$ matrices are sparse matrices that are related to the stored physical properties of each tetrahedron. In our case, we consider that $\mathbf{M}$ and $\mathbf{C}$ are diagonal matrices, i.e., that mass and damping effects are concentrated at vertices. This simplification called masslumping decouples the motion of all nodes and therefore allows us to write equation 10 as the set of independent differential equations for each vertex. 
Furthermore, we choose an explicit integration scheme where the elastic force is estimated at time $t$ in order to compute the vertex position at time $t+1$ :

$$
\left(\frac{m_{i}}{\Delta t^{2}}-\frac{\gamma_{i}}{2 \Delta t}\right) \mathbf{P}_{i}^{t+1}=\mathbf{F}_{i}+\frac{2 m_{i}}{\Delta t^{2}} \mathbf{P}_{i}^{t}-\left(\frac{m_{i}}{\Delta t^{2}}+\frac{\gamma_{i}}{2 \Delta t}\right) \mathbf{P}_{i}^{t-1}
$$

One of the basic tasks in surgery simulation consists in cutting soft tissue. With our deformable model, this task can be achieved efficiently. We simulate the action of an electric scalpel on soft tissue by successively removing tetrahedra at places where the instrument is in contact with the anatomical model.

When removing a tetrahedron, about a hundred update operations are performed to suppress the tetrahedron contributions to the stiffness data of the surrounding vertices, edges, and triangles. By locally updating stiffness data, the tissue has exactly the same properties as if we had removed the corresponding tetrahedron at its rest position. Because of the volumetric continuity of finite element modeling, the tissue deformation remains realistic during the cutting.

\section{Incompressibility Constraint}

Living tissue, which is essentially made of water, is nearly incompressible. This property is difficult to model and leads in most cases to instability problems. This is the case with the St Venant-Kirchhoff model: the material remains incompressible when the Lam constant $\lambda$ tends towards infinity. Taking a large value for $\lambda$ would force us to decrease the time step and therefore to increase the computation time. Another reason to add an external incompressibility constraint to our model is related to the model itself: the main advantage of the St Venant-Kirchhoff model is to use the strain tensor $E$ which is invariant with respect to rotations. But it is also invariant with respect to symmetries, which could lead to the reversal of some tetrahedra under strong constraints.

We choose to penalize volume variation by applying to each vertex of the tetrahedron a force directed along the normal of the opposite face $\mathbf{N}_{p}$ (see figure on the right), the norm of the force being the square of the relative volume variation:

$$
\mathbf{F}_{\text {incomp }}^{p}=\left(\frac{V-V_{0}}{V_{0}}\right)^{2} \mathbf{N}_{p}
$$

These forces act as an pressure increase inside the tetrahedron. This method is closely related to Lagrange multipliers, which are often used to solve problem of energy minimization under constraints.

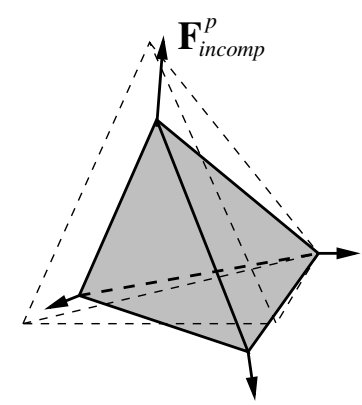

Fig. 4. Penalization of the volume variation

\section{Results}

In the first experiment, we wish to highlight the contributions of our new deformable model in the case of partial rotations. Figure 5 shows the same experi- 
ence as the one presented for linear elasticity (section 2.2, figure 3). On the left we can see that the cylinder vertices can now follow trajectories different from straight lines (figure 5(a)), leading to much more realistic deformations than in the linear (wire-frame) case (figures $5(\mathrm{~b})$ and $5(\mathrm{c})$ ).

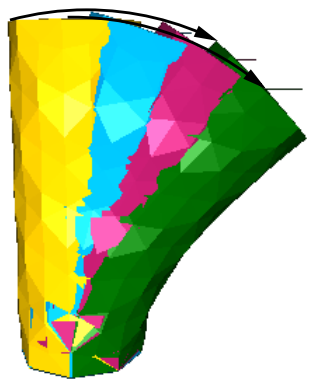

(a)

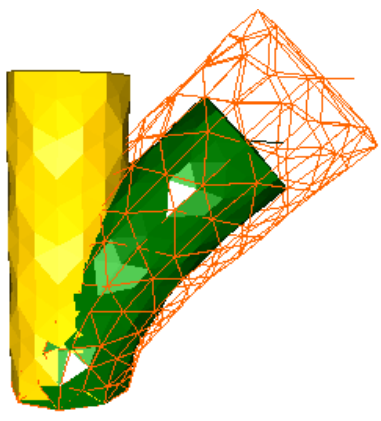

(b)

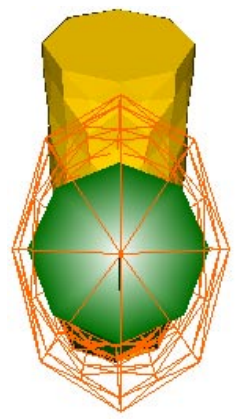

(c)

Fig. 5. (a) Successive deformations of the non linear model. Side (b) and top (c) view of the comparison between linear (wire-frame) and non linear model (solid rendering)

In the second example (figure 6), we apply a force to the right lobe of the liver (the liver is fixed on its central back part, and Lam constants are: $\lambda=4.10^{4}$ and $\left.\mu=10^{4}\right)$. Using the linear model, the right part of the liver undergoes a large (and unrealistic) volume increase, whereas with non-linear elasticity, the right lobe is able to rotate, giving a much more accurate deformation.
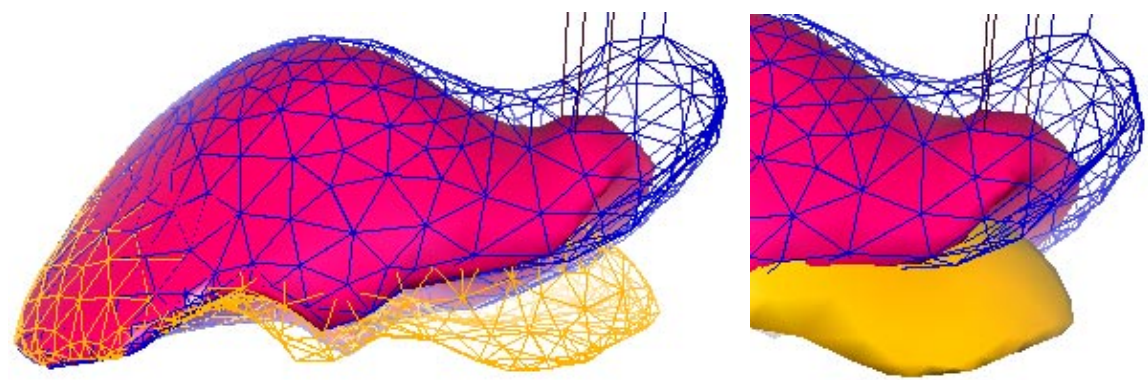

Fig. 6. Linear (wireframe), non-linear (solid) liver models, and rest shape (bottom)

Adding the incompressibility constraint on the same examples decreases the volume variation even more (see table $2^{1}$ ), and also stabilizes the behaviour of the deformable models in strongly constrained areas.

The last example is the simulation of a typical laparoscopic surgical gesture on the liver. One tool is pulling the edge of the liver sideways while a bipolar cautery device cuts it. During the cutting, the surgeon pulls away the part of

\footnotetext{
${ }^{1}$ For the cylinder: left, middle and right stand for the different deformations of figures 3 and 5(a)
} 


\begin{tabular}{|c|c|c|c|}
\hline Volume variations (\%) & Linear & Non-linear & Non-linear incomp. \\
\hline \begin{tabular}{|c|c|c|} 
Cylinder left & middle & right
\end{tabular} & \begin{tabular}{|l|l|l|}
7 & 28 & 63 \\
\end{tabular} & \begin{tabular}{l|l|l}
0.3 & 1 & 2
\end{tabular} & \begin{tabular}{l|l|l}
0.2 & 0.5 & 1 \\
\end{tabular} \\
\hline Liver & 9 & 1.5 & 0.7 \\
\hline
\end{tabular}

Table 2. Volume variation results

the liver he wants to remove. This piece of liver undergoes large displacements and the deformation appears fairly realistic with this new non-linear deformable model (figure 7).

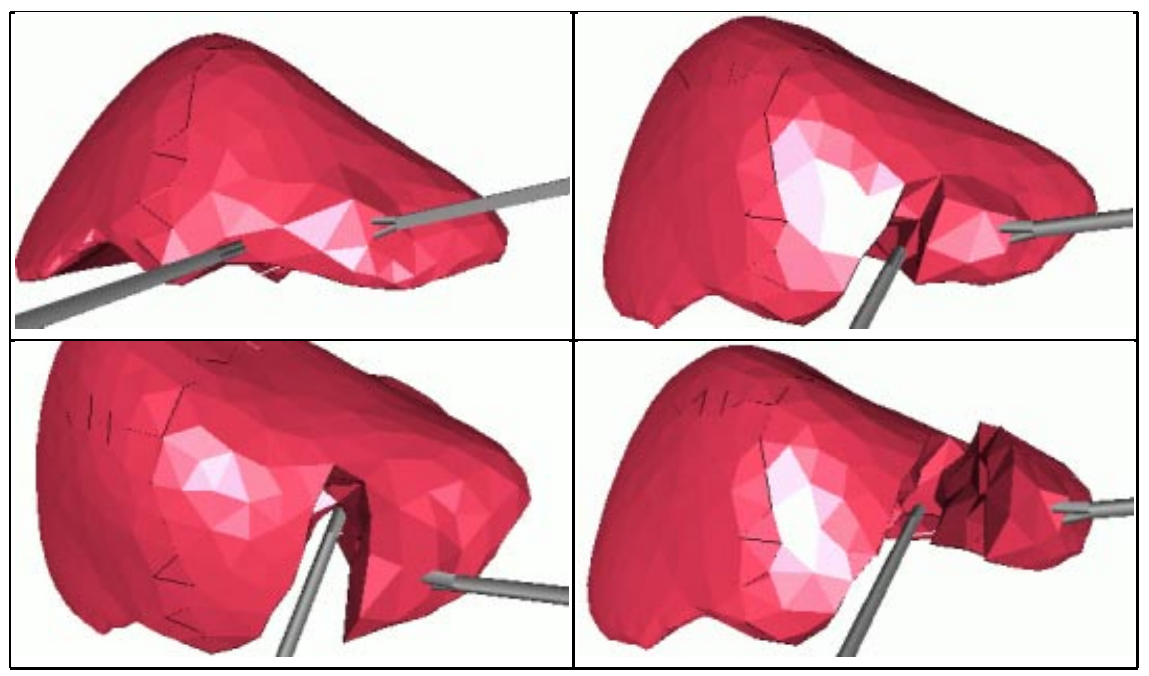

Fig. 7. Simulation of laparoscopic liver surgery

Obviously, the computation time of this model is larger than for the linear model because the force equation is much more complex (equation 8). With our current implementation, simulation frequency is four times slower than with the linear model. Nevertheless, with this non-linear model, we can reach a frequency update of $30 \mathrm{~Hz}$ on meshes made of about 2000 tetrahedra (on a PC Pentium PIII 500MHz). This is sufficient to reach visual real-time with quite complex objects, and even to provide a realistic haptic feedback using force extrapolation as described in [15].

\section{Conclusion}

We proposed in this article a new deformable model based on large displacement elasticity, a finite element method, and a dynamic explicit integration scheme. It solves the problem of rotational invariance and takes into account the incompressibility properties of biological tissues. Including this model into our laparoscopic surgery simulator prototype improves its bio-mechanical realism and thus increases its potential use for learning and training processes. 
Our future work will focus on generalizing large displacement elasticity to anisotropic materials. Also, the large displacement model requiring more computation time, it will be usefull to develop a "hybrid" model: linear elasticity for the strongly constrained parts of the organ and large displacement elasticity for the free parts that can undergo large deformations. This will be done following the experience we had with previous "hybrid" models of this type [7].

\section{References}

1. N. Ayache, S. Cotin, H. Delingette, J.-M. Clement, J. Marescaux, and M. Nord. Simulation of endoscopic surgery. Journal of Minimally Invasive Therapy and Allied Technologies (MITAT), 7(2):71-77, July 1998.

2. C. Basdogan, C. Ho, M. A. Srinivasan, S. D. Small, and S. L. Dawson. Force Interaction in Laparoscopic Simulation: Haptic Rendering Soft Tissues. In Medecine Meets Virtual Reality (MMVR'6), pages 28-31, San Diego CA, january 1998.

3. K.-L. Bathe. Finite Element Procedures in Engineering Analysis. Prentice-Hall, 1982.

4. Morten Bro-Nielsen and Stephane Cotin. Real-time Volumetric Deformable Models for Surgery Simulation using Finite Elements and Condensation. In Eurographics '96. ISSN 1067-7055, pages 57-66. Blackwell Publishers, 1996.

5. P. G. Ciarlet. Mathematical elasticity Vol. 1: Three-dimensional elasticity. Elsevier Science Publishers B.V., 1988.

6. S. Cotin, H. Delingette, and N. Ayache. Real-time elastic deformations of soft tissues for surgery simulation. IEEE Transactions On Visualization and Computer Graphics, 5(1):62-73, January-March 1999.

7. S. Cotin, H. Delingette, and N. Ayache. A Hybrid Elastic Model allowing RealTime Cutting, Deformations and Force-Feedback for Surgery Training and Simulation. The Visual Computer, 2000. to appear (see INRIA research report RR-3510).

8. F. Boux de Casson and C. Laugier. Modeling the Dynamics of a Human Liver for a Minimally Invasive Surgery Simulator. In MICCAI'99, Cambridge UK, 1999.

9. G. Debunne, M. Desbrun, A. Barr, and M.-P. Cani. Interactive multiresolution animation of deformable models. In 10th Eurographics Workshop on Computer Animation and Simulation (CAS'99), September 1999.

10. H. Delingette, S.Cotin, and N.Ayache. A Hybrid Elastic Model allowing Real-Time Cutting, Deformations and Force-Feedback for Surgery Training and Simulation. In Computer Animation, Geneva Switzerland, May 26-28 1999.

11. Y. C. Fung. Biomechanics - Mechanical Properties of Living Tissues. SpringerVerlag, second edition edition, 1993.

12. D. Lamy and C. Chaillou. Design, Implementation and Evaluation of an Haptic Interface for Surgical Gestures Training. In International Scientific Workshop on Virtual Reality and Prototyping, pages 107-116, Laval, France, June 1999.

13. J. Marescaux, J-M. Clément, V. Tassetti, C. Koehl, S. Cotin, Y. Russier, D. Mutter, H. Delingette, and N. Ayache. Virtual Reality Applied to Hepatic Surgery Simulation : The Next Revolution. Annals of Surgery, 228(5):627-634, 1998.

14. W. Maurel, Y. Wu, N. M. Thalmann, and D. Thalmann. Biomechanical Models for Soft Tissue Simulation. Springer-Verlag, 1998.

15. G. Picinbono, J.C. Lombardo, H. Delingette, and N. Ayache. Anisotropic Elasticity and Force Extrapolation to Improve Realism of Surgery Simulation. In IEEE International Conference on Robotics and Automotion: ICRA 2000, pages 596602, San Francisco, CA, April 2000. 